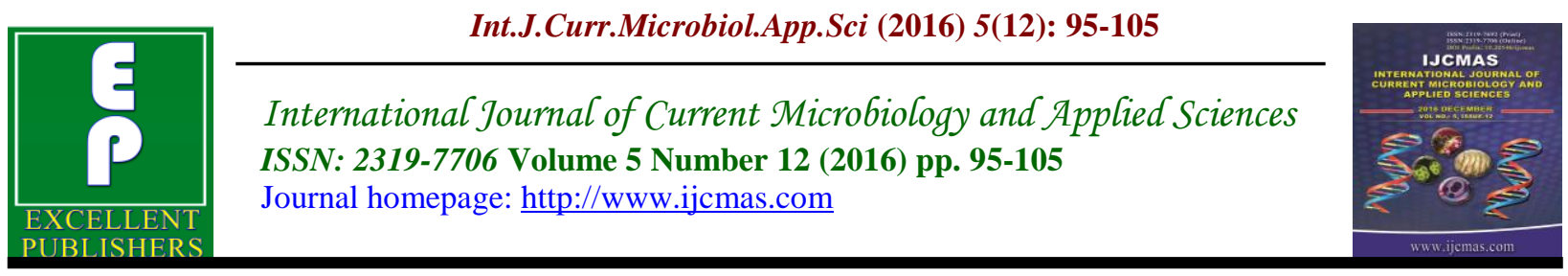

Original Research Article

http://dx.doi.org/10.20546/ijcmas.2016.512.011

\title{
Prevalence of Drug Resistant Gram Negative Bacilli (DRGNB) in Patients Suffering from Symptomatic Urinary Tract Infection from a Tertiary Care Hospital in Coimbatore, India
}

\author{
R.B. Varshini Narmada, R. Someshwaran" and Anbu N. Aravazhi \\ Department of Microbiology, Karpagam Faculty of Medical Sciences \& Research, \\ Othakalmandapam, Coimbatore-641032, India \\ *Corresponding author
}

Keywords

Antimicrobial chemotherapy Antibiotic policy Antibiotic Resistance - Culture and Sensitivity Infection control Urinary isolates.

Article Info

Accepted: 08 November 2016 Available Online: 10 December 2016

\section{A B S T R A C T}

Gram negative bacteria are highly adaptive pathogens that can develop resistance and evolve as Drug Resistant Gram Negative Bacilli (DR-GNB) by exhibiting Extended Spectrum Beta Lactamase (ESBLs), Amp C, NDM-1, Mcr-1 and Carbapenemase enzymes through several mechanisms. The main aim of this research was to study the prevalence of drug resistant gram negative bacilli in patients suffering from symptomatic urinary tract infections. After obtaining the IHEC clearance and informed consent form, nearly 820 urine samples were included in the study. Culture and sensitivity performed and various drug resistance patterns such as ESBLs, Amp C, K1 Enzymes, Carbapenemase production were detected and confirmed using various screening and confirmation tests. A total of 820 mid stream urine samples were collected from the subjects during the two months period. The mean age group of the study population was found to be $44.2 \pm$ 0.2 years. Out of the 820 urine samples processed for Culture and sensitivity, nearly 166 samples (20.2\%) grew bacteria and were termed 'Culture positives' and 654 samples $(79.7 \%)$ were sterile and were termed 'Culture negatives'. The incidence of Urinary tract infection was 20\%. ESBL production was observed in 17 out of 140 isolates $(12.14 \%)$. The antimicrobial resistance pattern is highly variable and a continuous surveillance of trends in resistance pattern is a borne necessity. To conclude Escherichia coli is the predominant pathogen isolated. The Prevalence of Drug resistant Gram Negative Bacilli thrusts a major therapeutic concern for the clinicians. Molecular method like Polymerase Chain Reaction is the Gold standard for confirmative diagnosis of these drug resistant bugs.

\section{Introduction}

Urinary tract infections are the second most common cause for Nosocomial infections accounting for $15 \%$ of the infections reported by acute care hospitals with a mortality of 15,000 deaths every year
(Klevens et al., 2002). Gram negative bacteria are known to be the main cause of Urinary tract infections (UTI). Escherichia coli is the predominant gram negative bacilli causing these infections responsible for 75 - 
$90 \%$ cases followed by gram negative bacilli Klebsiella pneumoniae and gram positive bacteria like Enterococcus faecalis, Streptococcus agalactiae, Bacillus subtilis (Savas et al., 2006). Women are more susceptible to UTI compared to men due to the female anatomy. Female urethra is much shorter and closer to the perineum (Moreno et al., 2008). Also decreased estrogen levels after menopause, increases the risk of acquiring UTI. Women who are sexually active, pregnant women, men with Chronic prostatitis, patients suffering from Diabetes mellitus type II, spinal cord injury requiring repeated and prolong catheterization are more prone to UTI (Lane et al., 2011). Nearly $80-85 \%$ of community acquired UTI are caused by Escherichia coli and 5-10\% by Staphylococcus saprophyticus. Other pathogens causing UTI are Klebsiella pneumoniae, Proteus spp., Enterobacter spp., Pseudomonas spp., Candida albicans, Enterococcus spp., and some viral infections are not so uncommon (Jaggi et al., 2012).

Various antibiotic agents have been developed to combat the severity and spread of infections. Among gram negative uropathogens the rate of resistance is high and frequency of resistance to antibiotics is directly related to consumption of antibiotics prescribed by physician and due to over the counter pharmacy prescriptions. Gram negative bacteria are highly adaptive pathogens that can develop resistance and evolve as Drug Resistant Gram Negative Bacilli (DR-GNB) by exhibiting Extended Spectrum Beta Lactamases (ESBLs), Amp C and Carbapenemase enzymes through several mechanisms. ESBLs are produced by Gram negative bacilli viz., Escherichia coli, Klebsiella pneumoniae, Enterobacter aerogenes, Citrobacter spp., Salmonella typhi, Shigella spp., Acinetobacter baumannii and Pseudomonas aeruginosa. Increasing antibiotic resistance is predominantly attributed to the prevalence of drug resistant nosocomial pathogen in the hospital environment and also due to the negligence of the medical practitioners to an extent where prescription of antibiotics do happen in an inappropriate or irrational fashion (Drekonja et al., 2008). Klebsiella pneumoniae Carbapenemase (KPC) are more common nowadays rendering beta lactams and Carbapenems resistant and the physicians are left with minimal or nearly no appropriate antibiotics of choice. K1 enzyme is one more type of drug resistance encountered in Klebsiella oxytoca.

Community acquired urinary tract infections are treated with Cotrimoxazole generally. Hospital acquired urinary tract infections are treated with first or second generation Cephalosporins. But E.coli have the inherent potential to produce drug resistance by acquisition of Extended Spectrum Beta Lactamase (ESBL) enzyme, which is capable of degrading beta lactam antibiotics like Penicillins and Extended Spectrum Cephalosporins by hydrolysis of betalactam ring (Borus et al., 2010). Drug resistant E.coli strains if left untreated may eventually pose an abysma threat, associated with development of co-morbidities and profound mortality rates. Such cases are advised to be managed by Nitrofurantoin, Fluoroquinolones, Carbapenem, Fosfomycin, Mecillinam, Rifampicin, Colistin or Tigecycline. Most bacilli with Amp C enzyme showed resistance to third generation Cephalosporins, Aztreonam, and beta lactamase inhibitors, but susceptible to Imipenem, Cefepime, Meropenem etc. Klebsiella pneumoniae are known to produce specific drug resistance enzyme namely Klebsiella pneumoniae Carbapenemase enzyme (KPC) conferring resistance to broad spectrum antibiotics like Penicillins, Cephalosporins and also carbapenems (Yong et al., 2002). 
Tigecycline, Colistin and Rifampicin are the treatment options for Carbapenemase producing Gram Negative Bacilli. Specific drug resistance patterns like New Delhi Beta lactamases (NDM-1), and Pan drug resistant Escherichia coli due to the gene Mcr-1 are some super bugs who had caused a recent turmoil in infections control management of health care associated infections.

The main of this study the prevalence of Drug Resistant Gram Negative Bacilli in patients suffering from symptomatic urinary tract infection. The main objective of this study is to isolate and identify Gram Negative Bacilli in urine by routine culture methods. To evaluate Antibiotic Susceptibility Test pattern of these isolates by Kirby Bauer's method. And also to detect drug resistance patterns like ESBLs, Amp C, $\mathrm{K} 1$ enzyme, and Carbapenemase production among Gram negative bacilli in urinary isolates.

\section{Inclusion criteria}

In-patients and out- patients with comorbid conditions like Diabetes mellitus, Spinal cord injury, patients requiring Long term catheterization, Pregnancy and etc., with clinical features of urinary tract infections. In-patients and Out-patients of Medicine, Surgery, Obstetrics and Gynecology, and Orthopedics departments, clinically diagnosed as Urinary tract infection by the treating physician.

Age group: Between 18 years to 60 years of age.

\section{Exclusion criteria}

Patient who had received antibiotics in last 1 week.

Patient not willing to give informed written consent.

Pediatric population <18 years of age.

\section{Materials and Methods}

A Cross sectional study was undertaken after clearance from Institutional Human Ethics Committee (IHEC). Informed written consent was obtained from the study participants viz., In-patients and outpatients clinically diagnosed to have urinary tract infections from Medicine, Orthopedics and Surgery departments. A total of 820 urine samples that were received in the Microbiological laboratory during the study period were included for this research study. Under sterile precautions, mid-stream urine (MSU) samples were collected in a sterile leak proof wide mouthed plastic container from the patients prior to initiation of antibiotic therapy (Winokur et al., 2010). Urine samples after collection were transported immediately to the Microbiology laboratory (within 30 minutes) and were further processed by performing routine diagnostic tests like Urine wet mount preparation, Aerobic culture, Appropriate biochemical tests to aid in bacterial identification and the Antibiotic Susceptibility Testing were done and different sensitivity pattern observed, analyzed and recorded as per Standard Operating Procedures. Urine samples if not processed immediately were refrigerated at $4-8^{\circ} \mathrm{C}$ but further processed within two hours of storage. A direct uncentrifuged urine wet mount preparation was made and visualized under light microscopy (40X objective) for presence of pus cells, bacteria, RBC's, Yeast cells and for parasites if any. Semi- quantitative urine cultures were done with $5 \mu \mathrm{L}$ volume in a $2 \mathrm{~mm}$ calibrated sterile small sized loop on routine plating media like Blood Agar Plate (BAP) and Mac Conkey Agar Plate (MAP) and were then incubated aerobically at $37^{\circ} \mathrm{C}$ for 24 hours. Counts of $\geq 10^{5}$ Colony Forming Units per milliliter $(\mathrm{CFU} / \mathrm{mL})$ of urine were considered as "Significant bacteriuria". Gram stain films of bacterial colonies 
isolated on solid culture media were observed under light microscopy for presence of Gram negative bacilli (Hsu et al., 2011). The culture isolates were identified by routine methods as per standard guidelines by interpretation of colonial morphology, Biochemical reactions and some special tests like catalase, oxidase, nitrate, motility and etc.

Antibiotic Susceptibility Test (AST) were done on Mueller-Hinton Agar using by Kirby Bauer's Disk diffusion method with 0.5 Mac Farland turbidity standard of gram negative bacterial suspension according to Clinical and Laboratory Standards Institute (CLSI) 2016 guidelines. A lawn culture of test organism were made over Mueller Hinton Agar Plate (MHAP) and different antibiotics to be tested for susceptibility were placed as individual commercially available antibiotic discs (Procured from Hi Media) of fixed antibiotic concentrations.

MHAP were incubated at $37^{\circ} \mathrm{C}$ under aerobic incubation for a period of 16-24 hours and Antibiotic Susceptibility Patterns were noted by reading the zone of inhibition over around the antibiotic disc (Silva et al., 2000).

Quality control for culture and AST were done using standard reference strains like Escherichia coli (ESBL Negative) ATCC 25922 as per EUCAST and or CLSI 2016 guidelines. In this study, Ten Disk procedure were used to detect various Drug resistance viz., ESBL, Amp C, K1 enzyme and Carbapenemase production and further confirmed by appropriate Combination Disk or Double Disk Potentiation methods as per
CLSI. Modified Hodge tests were performed for suspected Carbapenemase producers. Specific Antibiotic Drug Resistance Patterns like Extended Spectrum Beta Lactamases, Amp C production, K1 enzyme and Carbapenemase production were detected as per Center for Laboratory Standard Institute (CLSI) 2016 and or European Committee on Antimicrobial Susceptibility Testing (EUCAST) guidelines by the following methods.

\section{ESBL detection (Screening Test)}

According to EUCAST and CLSI guidelines, Ceftazidime, Cefotaxime and Cefpodoxime were used to perform disk diffusion method of screening for ESBL production. A zone difference of $\geq 5 \mathrm{~mm}$ in Ceftazidime (CAZ) and Ceftazidime Clavulanic acid (CAZ-CLA) discs is suggestive of possible ESBL production as per CLSI guidelines. If screening test was positive, phenotypic Confirmation methods are used to diagnose ESBL.

\section{ESBL detection (Confirmatory Test)}

A zone difference of $\geq 5 \mathrm{~mm}$ between Cefotaxime (CTX) and Cefotaxime Clavulanic acid (CTX- CLA) with Key hole or clavulanate effect was confirmatory for ESBL production as per CLSI guidelines. A Double Disk Synergy Test (DDST) and Direct modified Three Dimensional Test (DMTDT) were done to detect ESBL. ESBL positive isolates were reported as resistant to Beta lactam antibiotics like Penicillins, Cephalosporins and Monobactums. ESBL production among Amp $\mathrm{C}$ positive isolates were done by Modified Double Disk Synergy Test (MDDST). 
ESBL SCREENING

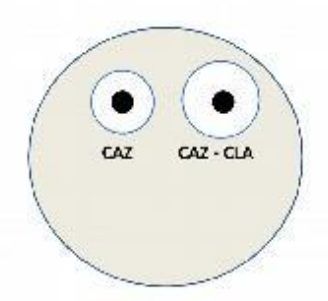

ESBL CONFIRMATION

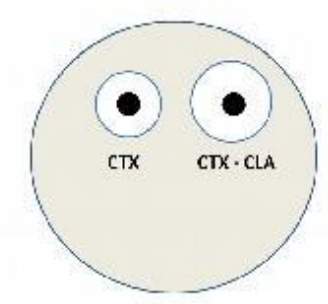

\section{Amp C detection (Screening Test)}

According to CLSI guidelines, Cephamycins like Cefoxitin or Cefotetan are used to detect probable Amp C. Cefoxitin resistance (Zone diameter $\leq 18 \mathrm{~mm}$ ) is considered as a marker for probable Amp C production.

\section{Amp C detection (confirmatory test)}

Phenotypic confirmatory test used to diagnose Amp C production is detected by Double Disc Synergy Test (DDST) using Cefoxitin $(30 \mu \mathrm{g})$ and Cloxacillin $(200 \mu \mathrm{g})$ : This test is based on the inhibitory effect of Cloxacillin on Amp $\mathrm{C}$ the strains are inoculated in Mueller Hinton agar. A difference in Cefoxitin- cloxacillin inhibition zones minus cefoxitin alone zones of $>4 \mathrm{~mm}$ was considered indicative for Amp C production (Yong et al., 2002). A Modified Three Dimensional test (MTDT) were used to detect Amp C Beta Lactamase in ESBL positive isolateswith reduced susceptibility to Cefoxitin $(\leq 18 \mathrm{~mm})$ (Khan et al., 2007).
ESBL SCREENING

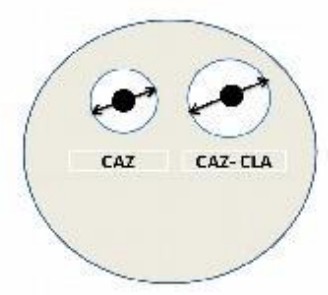

ESBL CONFIRMATION

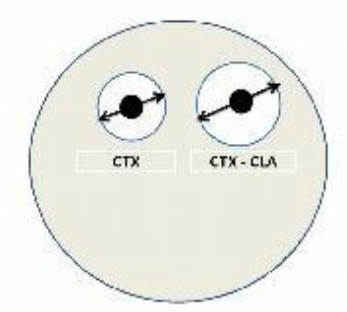

Detection of K1 enzyme Betalactamase
for Klebsiella oxytoca

Presence of Aztreonam resistance and Ceftazidime sensitivity with no clavulanic effect is observed to diagnose K1 enzyme beta lactamase.

\section{Carbapenemase Detection (Screening and Confirmatory tests)}

Carbapenemase screening is done by using Imipenem, Ertapenem and or Meropenem discs. If either or all of the Carbapenems are Resistant by Disc diffusion method then Modified Hodge Test (MHT) is done to confirm Carbapenemase production. A lawn culture of Escherichia coli ATCC 25922 is done on Mueller Hinton Agar Plate and the Carbapenem disc is placed in the center of the plate and the plate is incubated at $37^{\circ} \mathrm{C}$ for 24 hours under aerobic incubation and the next day the results are noted.

The test isolate, positive control and negative control are inoculated in a straight 
line from the edge of carbapenem disk to edge of the MHAP. Clover-Leaf pattern of indentation (Club shaped) at the intersection of the test organism and the ATCC strain

\section{CARBAPENEMASE DETECTION}

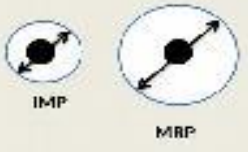

Evaluation of Minimum Inhibitory Concentration (MIC break point) for antibiotics is so specific for determining antibiotic resistance and is to be determined by Broth micro-dilution method, Agar dilution method, Epsilometric test (Etest) method and or by using automated systems like Vitek-2 Identification system.

\section{Results and Discussion}

A total of 820 mid stream urine samples were collected from the subjects during the two months period. Female preponderance was observed in the study population selected based on inclusion criteria where 594 cases $(72.44 \%)$ were females and remaining 226 cases $(27.56 \%)$ were males. The mean age group of the study population was found to be $44.2 \pm 0.2$ years. Out of the 820 urine samples processed for Culture and sensitivity, nearly 166 samples (20.2\%) grew bacteria and were termed 'Culture positives' and 654 samples $(79.7 \%)$ were sterile and were termed 'Culture negatives'. Among Culture Positives 140 out of 166 (84.34\%) Culture positive isolates were identified as Gram Negative Bacilli viz., Escherichia coli (98 out of 140, 70\%), Enterobacter spp., (19 out of 140, 13.57\%), Klebsiella spp., (14 out of 140, 10\%), Proteus spp., (8 out of $140,5.71 \%$ ) and within the zone of inhibition" which is confirmatory for Carbapenemase enzyme production by (Mathew, 2014).

\section{MODIFIED HODGE TEST}

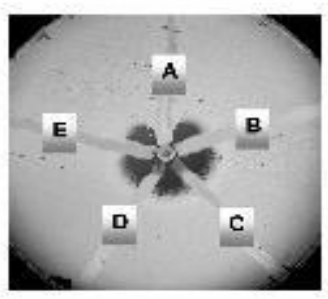

Pseudomonas aeruginosa (1 out of 140, $0.71 \%$ ). The predominant isolate observed among Gram Negative Bacteria was Escherichia coli followed by Enterobacter spp., and Klebsiella spp. And the remaining 26 out of 166 (15.66\%) Culture positive isolates were identified as Gram Positive cocci viz., MSSA (5 Out of 26, 19.23\%), MRSA (1 Out of 26, 3.84\%), MRCONS (4 Out of 26, 15.38\%), MSCONS (9 Out of 26, $34.61 \%)$, Staphylococcus saprophyticus (1 Out of 26, 3.8\%0), Enterococcus faecium (5 Out of 26, 19.23\%) and Enterococcus fecalis (1 Out of $26,3.84 \%$ ). The predominant Gram positive cocci that was isolated among study population were Methicillin Sensitive Coagulase Negative Staphylococci (MSCONS) followed by Methicillin Sensitive Staphylococcus aureus (MSSA) and Enterococcus faecium respectively. The specific drug resistance of gram negative bacilli was identified by the screening the culture isolates for production of ESBL, Amp C, K1 enzyme and Carbapenemase beta lactamase enzymes. ESBL production was observed in 17 out of 140 isolates (12.14\%). Escherichia coli ESBL production rate was observed to be 13 out of 98 isolates (13.26\%). ESBL producing Klebsiella pneumoniae, Enterobacter aerogenes, and Proteus spp., were found to be 2 out of 11(18.18\%), 1 out of 8 (12.5\%) 
and 1 out of $8(12.5 \%)$. Inducible AmpC production was observed in 4 out of 98 $(\approx 4 \%)$ Escherichia coli isolates and also in 2 out of 14 (14.28\%) of the Klebsiella spp. No $\mathrm{K} 1$ enzyme production was detected among 3 of the Klebsiella oxytoca isolates. Among these 140 Gram Negative Bacilli isolated Carbapenemase production was observed only in Klebsiella pneumoniae. As only 1 out of 11 (9.09\%) Klebsiella pneumoniae isolates was found to be Carbapenemase producer (Klebsiella pneumoniae Carbapenemase - KPC) which was confirmed by Modified Hodge Test.

Monoresistance viz., Ampicillin resistance was observed in 102 out of $140(72.85 \%)$ cases. Ciprofloxacin resistance was observed in 97 out of 140 (69.28\%) isolates. Cotrimoxazole and Nitrofurantoin resistance were observed in 81 out of $140(57.85 \%)$ and 93 out of $140(66.42 \%)$ of the Gram negative isolates. Carbapenem had the highest sensitivity of $99.28 \%$ in 139 out of 140 bacterial isolates. Among Escherichia coli isolates 66 out of $98(67.34 \%), 72$ out of $98(73.48 \%), 81$ out of $98(82.65 \%), 80$ out of $98(81.63 \%)$ and 98 out of $98(100 \%)$ isolates were sensitive to Nitrofurantoin, Piperacillin Tazobactam, Amikacin, Cefoperazone Sulbactam and Imipenem respectively. ESBL coupled AmpC production was observed in 2 out of 98 $(\approx 2 \%)$ isolates of Escherichia coli isolates. Enterobacter isolates were mostly sensitive to Piperacillin Tazobactam, Amikacin, Ceftriaxone, Cefoperazone sulbactam and Nitrofurantoin. Klebsiella spp., showed $100 \%$ resistance to ampicillin. One isolate identified as KPC were found to be resistant to nearly all antibiotics except Tigecyline, Colistin and Amikacin.

In this study, the study population had moderate female preponderance where females consisted of $72.4 \%$ of the study population. Out of a total of $820 \mathrm{Mid}$-stream urine samples obtained for culture and sensitivity, $166 \quad(20.2 \%)$ were culture positive. The incidence of Urinary tract infection was 20\%. Among the culture positive isolates114 out of $166(68.67 \%)$ belonged to female patients which proves the point that UTI's are more common in female patients which was similar to a study done which showed that females are more susceptible than males to UTI. And among Culture positive cases, 140 out of 166 $(84.33 \%)$ Culture positive isolates were found to be Gram Negative Bacilli (GNB) by wet mount preparation, culture, colony Gram stain and other biochemical tests. The incidence of Gram negative Urinary Tract Infection (UTI) was found to be $\approx 84 \%$. Although Escherichia coli was the most frequent and predominant pathogen isolated among symptomatic UTI cases, significant presence of other urinary pathogens like Klebsiella spp., and Enterobacter spp., were also observed. Among Gram negative bacilli isolated Escherichia coli were isolated in 98 out of 140 cases making the incidence of Escherichia coli as 70\%. Incidence of Enterobacter spp., and Klebsiella spp., were found to be $13.57 \%$ and $10 \%$ respectively. ESBL production was found in 18 out of 140 isolates making it $12.85 \%$ among the Gram Negative Bacilli isolates. The incidence of ESBL producing Escherichia coli was found to be $13.26 \%$ which is found to be less compared to some research studies done elsewhere who had reported a higher prevalence of up to $65-80 \%$ (Colander et al., 2001). These ESBL producing Escherichia coli isolates were found to be mostly sensitive to Imipenem, Piperacillin Tazobactam and Amikacin. A study done in North India had more less similar sensitivity pattern as obtained in this study. Ampicillin resistance was observed in $72.85 \%$ cases followed by Ciprofloxacin resistance which was observed $69.28 \%$ isolates. Cotrimoxazole and Nitrofurantoin resistance 
were observed in $57.85 \%$ and $66.42 \%$ of the cases which grew Gram negative isolates. Carbapenems had the highest sensitivity of 99.28\% among GNB. The sensitivity of Nitrofurantoin, Piperacillin Tazobactam, Amikacin, Cefoperazone Sulbactam and Imipenem were found to be $67.34 \%$, $73.48 \%, 82.65 \%, 81.63 \%$ and $100 \%$ respectively. The potentiation of ESBL coupled AmpC production was observed in 2 out of $98(\approx 2 \%)$ isolates of Escherichia coli isolates. Enterobacter isolates were mostly sensitive to Piperacillin Tazobactam, Amikacin, Ceftriaxone, Cefoperazone sulbactam and Nitrofurantoin. Klebsiella spp., showed $100 \%$ resistance to ampicillin. Klebsiella pneumoniae carbapenemase (KPC) was found to be sensitive only to Tigecyline, Colistin and Amikacin. Overall incidence of ESBL, AmpC and Carbapenemase producers among 140 Gram Negative Bacteria were found to be $12.85 \%$, $4.2 \%$ and $0.7 \%$ respectively. Antibiotic resistance has become a major issue which threatens the public health nowadays and it is alarming to note that almost all isolates included in this study were found resistant to a minimum of two or more antibiotics.

Nowadays Drug resistant Gram Negative Bacilli is more common in Hospitals and it thrusts a significant threat by leaving the physician with limited treatment options like availability of sensitive antibiotics to treat the same increasing the morbidity and mortality and also by increasing the duration of stay of the patient, cost burden etc. Irrational antibiotic misuse is the main cause for antibiotic resistance and surveillance measures to know the prevalence of DRGNB in our hospital is a borne necessity. Infection control committee should effectively formulate and update the Hospital Antibiotic Policy for to provide physicians a better understanding of the hospital strains and also for the better therapeutic management of the drug resistant bugs. To ensure the importance of hospital infection Control Committee (HICC) and its role in preventing Nosocomial infections. Continuous scrutiny or screening for Drug resistant Gram Negative Bacilli (DR-GNB) especially ESBL, AmpC, Carbapenemase producers among cases of urinary tract infection is mandatory. Following stringent Sterilization \& Disinfection and or Hospital Infection Control practices while providing patient care with the support of effective CSSD and HICC. Isolating subjects harboring Carbapenemase is necessary and providing them with early specific therapy as per AST report will possibly prevent the spread of infection. Hand washing should be strictly followed before, during and after patient care by doctors and nurses. Creating awareness and imparting knowledge about drug resistant bugs like ESBL, Amp C and Carbapenemase is necessary for primordial prevention of these drug resistant bugs causing Nosocomial infections or Health care associated infections (HAI's). Periodic Medical audits on right antibiotic prescription as per Institutional antibiotic policy must be regularly carried out in order to prevent 'iatrogenic induction' of these drug resistant bugs and further prevent a susceptible bacteria becoming drug resistant in the future.

\section{Limitations of the study}

The limitations of the study were a) Getting consent for the study b) Plate Contamination while performing culture and sensitivity hindered the interpretation and delayed the issue of the results; c) As this is a crosssectional study, the subjects who harbored these DRGNB the follow up was not done to ensure the appropriate antibiotic prescription, this could be overcome by conducting regular medical prescription audits. 
Table.1 Specific Drug Resistance Pattern among Gram Negative Bacilli Isolated In Urine

\begin{tabular}{|l|l|c|c|c|c|c|}
\hline S. & Bacterial isolate & No. of isolates & \multicolumn{4}{|l|}{ SPECIFIC DRUG RESISTANCE PATTERN } \\
\cline { 4 - 7 } No & & & ESBL & AmpC & $\begin{array}{l}\text { K1 } \\
\text { Enzyme }\end{array}$ & Carbapenemase \\
\hline $\mathbf{1}$ & Escherichia coli & $\mathbf{9 8}$ & $\mathbf{1 3}$ & $\mathbf{4}$ & - & $\mathbf{0}$ \\
\hline $\mathbf{2}$ & Enterobacter aerogenes & $\mathbf{8}$ & $\mathbf{1}$ & $\mathbf{0}$ & - & $\mathbf{0}$ \\
\hline $\mathbf{3}$ & Enterobacter cloacae & $\mathbf{1 1}$ & $\mathbf{0}$ & $\mathbf{0}$ & - & $\mathbf{0}$ \\
\hline $\mathbf{4}$ & Klebsiella pneumoniae & $\mathbf{1 1}$ & $\mathbf{2}$ & $\mathbf{2}$ & - & $\mathbf{1}$ \\
\hline $\mathbf{5}$ & Proteus vulgaris & $\mathbf{6}$ & $\mathbf{1}$ & $\mathbf{0}$ & - & $\mathbf{0}$ \\
\hline $\mathbf{6}$ & Klebsiella oxytoca & $\mathbf{3}$ & $\mathbf{0}$ & $\mathbf{0}$ & $\mathbf{0}$ & $\mathbf{0}$ \\
\hline $\mathbf{7}$ & Proteus mirabilis & $\mathbf{2}$ & $\mathbf{0}$ & $\mathbf{0}$ & - & $\mathbf{0}$ \\
\hline $\mathbf{8}$ & Pseudomonas aeruginosa & $\mathbf{1}$ & $\mathbf{0}$ & $\mathbf{0}$ & - & $\mathbf{0}$ \\
\hline & TOTAL NUMBER & $\mathbf{1 4 0}$ & $\mathbf{1 7}$ & $\mathbf{6}$ & $\mathbf{0}$ & $\mathbf{1}$ \\
\hline
\end{tabular}

Fig.1 Ten disk procedure to detect ESBL and Amp C and Carbapenemase

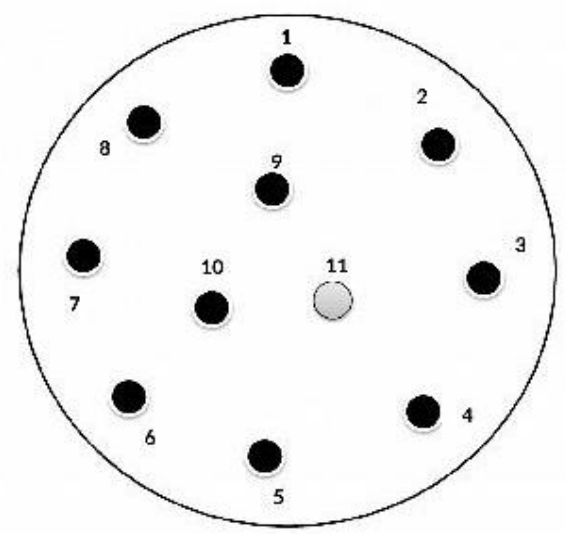

Fig.2 Distribution of Gram Negative Bacilli among Culture positive isolates

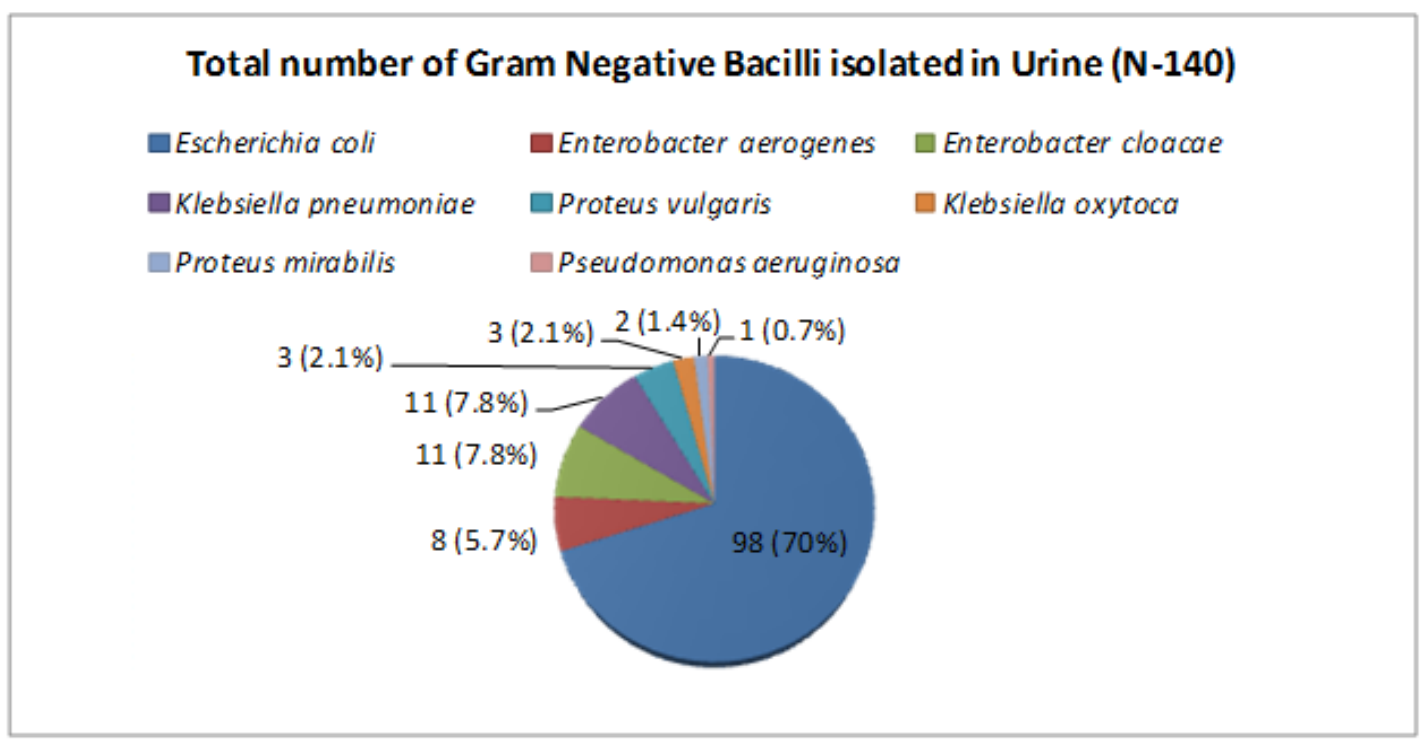

D) Budget was one more limitation which restricted us from carrying out molecular studies for further detection of specific drug resistance genes and for further confirmation 
of the DRGNB. This can be carried out as Phase II project in the department later.

To conclude Escherichia coli is the predominant pathogen isolated in this study. The Prevalence of Drug resistant Gram Negative Bacilli thrusts a major therapeutic concern for the clinicians. The antimicrobial resistance pattern is highly variable and a continuous surveillance of trends in resistance pattern is a borne necessity. Molecular method like Polymerase Chain Reaction (PCR) prevails to be the Gold standard for confirmative diagnosis of the drug resistant bugs.

\section{Acknowledgement}

We wish to thank the Dean and the management for the permission, support and constant encouragement rendered throughout the study. We also extend our thanks to Indian Council of Medical Research (ICMR) for its approval and acceptance of this research project as this work was done as a part of ICMR Short Term Studentship (STS) - 2016.

\section{References}

Borus, P.H.A., Polak, R., Hopemlman, A.I.M., Delgado, E. 2010. Jarquin increasing resistance in community acquired UTI, 2010; 14: e770-e774.

Colander, R., Keness, Y., chazzan, B., Raz, R. 2001. Antimicrobial susceptibility of community-acquired oropathogens in northern isreal. Int. J. Antimicrob. Agents, 18: 189-192. 10.1016/S09248579(01)00368-5.

Diurex, I., Brossier, F., Soughakoff, Jarlier, V. 2008. Phenotypic detection of ESBL, 14:90- 103.

Drekonja, D.M., Jhonson. 2008. Urinary tract infections. Prim. Care,345-367.
Dubey, D., REath, S., Sahu, M.C., padhy, R.S. 2010. Culture methods for gram negative bacilli, 13: 766-789.

Forbes, B.A., Sahm, D.F., Wiessefeild, A.S. 2007. Bailey \& Scotts Diagnostic microbiology. 12 editions. St. Louis mosbly Elsevier.

Hsu, L.Y., Tan, T.Y., Tam, V.H., Kwa, A., Fischer, D.A., Koh, D.H. et al. 2010. Pure culture production including gram negative bacilli, 14615-622.

Jaggi, N., Sissodia, P., Sharma, L. 2012. Control of multidrug resistant bacteria in tertiary care hospital in India. Antimicrob Resistant Infect. Control, 1: 23.

Kahlmeter, G. 2003. Prevalence and antimicrobial susceptibility of pathogens in uncomplicated cystitis Europe. The eco sens study. Int. J. Antimicrob. Agents, 22: 49-52. 10.1016/s0924-8579(03)00229-2.

Khan, M.K.R., S. Thukral, R. Gaind. 2008. Evaluation of a Modified DoubleDisc Synergy Test for Detection of Extended Spectrum B-Lactamases in Ampc B-Lactamase Producing Proteus mirabilis. Indian J. Med. Microbiol., Pg. 58-61.

Klevens, R.M., Edward, J.R., et al. 2007. Estimating health careassociated infections and deaths in U.S. hospitals, 2002. Public Health Reports, 122: 160-166.

Lane, D.R., Thakkar, S.S. 2011. Diagnosis and management of urinary tract infections, Emerg. Med. Clin. North Am., 29: 539-552.

Mathew, E., Falagas, Panagiota Lourida, Panagiotis Poulikakos, Petros, I., Rafailidis, Giannoula, S., Tansarli. 2014. Antimicrob. Agents Chemother., 58(2): 654-663.

Moreno, E., Andrew, A., Pigaru, C., Kuskowski, M.A. 2006. Acute cystitis 
in women, J. Clin. Microbial., 46: 2529-2534.

Sahu, M.C., Rath, S., Dubey, D. Debata, N.K., Padhy. 2012. Antibiotic susceptibility test to determine resistance of bacilli, 16: 322-313.

Savas, L., Guvel, S., Savas, N., Duran, N. 2006. Nosocomial urinary tract infections: micro-organisms, antibiotic sensitivities and risk factors. West Indian Med. J., 55: 188-193.

Silva, J., Aguliar, C., Ayala, g., Esterda, M.A., Garza ramos, U., Lara Lemus, R., et al. 2000. TLA-1: a new plasmid mediated extended spectrum beta lactamase from E.Coli ${ }_{3} 44:$ 997-1003.
Winokur, P.L.A. Bruegggemann, D.L. DeSalvo, L. 2010. Hoffman. Antimicrobial drug resistant carbanemase enzyme resistance, 13: 334-399.

Yong, D., R. Park. J. Yum, K. Lee, E. Choi. 2002. Further modification of hodge test to screen Amp-C producing strains, 51: 407.

Yong, D.R., Park, J. yum, Lee. 2002. Further modifications to screen Amp C beta lactramase producing strains, 52:407.

\section{How to cite this article:}

Varshini Narmada, R.B., R. Someshwaran and Anbu N. Aravazhi. 2016. Prevalence of Drug Resistant Gram Negative Bacilli (DRGNB) in Patients Suffering from Symptomatic Urinary Tract Infection from a Tertiary Care Hospital in Coimbatore, India. Int.J.Curr.Microbiol.App.Sci. 5(12): 95-105. doi: http://dx.doi.org/10.20546/ijcmas.2016.512.011 